\title{
O USO DE BADGES PARA PROMOVER A MOTIVAÇÃO E O ENGAJAMENTO DE DISCENTES NA DISCIPLINA ONLINE DE METODOLOGIA DA PESQUISA DO ENSINO SUPERIOR
}

\author{
CURITIBA/PR MAIO/2018
}

\author{
Patrícia da Silva Tristão - FAE/UNINTER - patriciatristao9@gmail.com \\ João Augusto Mattar Netto - UNINTER/PUC-SP - joaomattar@gmail.com \\ Eduardo Stumpf - FAE - eduardo.stumpf@fae.edu \\ Alvino Moser - UNINTER - moseral.am@gmail.com
}

Tipo: Investigação Científica (IC)

Natureza: Descrição de Projeto em Andamento

Categoria: Métodos e Tecnologias

Setor Educacional: EDUCAÇÃO SUPERIOR

\section{RESUMO}

Este artigo descreve uma pesquisa em andamento de abordagem qualitativa do tipo estudo de caso, que está sendo realizada com discentes do ensino superior de uma IES particular, matriculados na disciplina de Metodologia da Pesquisa na modalidade a distância. A pesquisa em questão tem o objetivo de analisar a contribuição do uso de badges como recompensa para promover a motivação e o engajamento dos alunos durante a disciplina de metodologia da pesquisa. A investigação envolve uma turma com 90 alunos de diferentes cursos de graduação. Os dados serão coletados a partir de um questionário de perguntas abertas e fechadas com os alunos, entrevista com a tutora da disciplina e análise dos relatórios do ambiente virtual de aprendizagem. Espera-se que os resultados da pesquisa revelem que o uso de badges aumenta a motivação e o engajamento dos alunos. Este artigo descreve o design da disciplina e a construção dos badges.

Palavras-chave: Ensino Superior. Educação a Distância. Metodologia da Pesquisa. Gamificação. 


\section{Introdução}

Este trabalho apresenta uma pesquisa em andamento sobre o uso de badges na disciplina de Metodologia da Pesquisa do Ensino Superior na modalidade EaD.

É conhecida a dificuldade dos discentes em cursar a disciplina de metodologia da pesquisa (SILLAOTS, 2014a, 2014b), requisito fundamental para a obtenção do diploma do ensino superior. Desta forma, foi implementado um estudo de caso com o objetivo de aplicar badges como recompensa para promover a motivação e engajamento dos alunos durante a disciplina de metodologia de pesquisa no nível da graduação, ofertada na modalidade da educação à distância. A proposta ainda apresenta como objetivos específicos: analisar o impacto e possíveis contribuições do uso de badges para o engajamento e motivação dos discentes para o estudo de Metodologia da Pesquisa.

Por meio de revisão bibliográfica, são analisados os conceitos que envolvem a gamificação e como ela pode contribuir para o engajamento e motivação de discentes na aprendizagem da metodologia da pesquisa, o uso de badges como recompensa para pequenas conquistas e a relação dos alunos com a disciplina de metodologia científica. Para tanto, o referencial teórico adotado compreende Deterding et al (2011), Kapp (2012) e Mattar (2014). Também são estudadas pesquisas mais recentes sobre o tema, como as de França (2016), Klock (2017), Landers (2014) e Sillaots (2014a, 2014b).

Para atingir os objetivos apresentados, a investigação se dá por meio de pesquisa aplicada de abordagem qualitativa e quantitativa, experimental, e por se tratar de um fenômeno contemporâneo, é delineada como estudo de caso (YIN, 2015). A população pesquisada compreende 90 discentes matriculados na disciplina de Metodologia da Pesquisa de uma IES em Curitiba e o professor-tutor.

Serão adotados quatro tipos de procedimentos para a coleta de dados: observações qualitativas e não participante durante o período da disciplina, pesquisa documental, entrevista semiestrututrada com o professor-tutor da disciplina e questionário aplicado aos alunos.

Este artigo apresenta o percurso de desenvolvimento dos badges, além do referencial teórico que embasa o projeto.

\section{Gamificação}

A gamificação, definida por Deterding et al (2011, tradução dos autores) como o "uso de 
elementos de videogames em contextos de não games para promover a experiência e o engajamento do usuário", tem conquistando espaços em diversos âmbitos, como, por exemplo, na gestão de pessoas, em aplicativos de saúde e de ensino de idiomas para motivar e envolver as pessoas.

$\mathrm{Na}$ educação, a gamificação pode ser adotada como uma estratégia de aprendizagem almejando o engajamento dos alunos em cursos e disciplinas, tanto presenciais quanto à distância.

Kapp (2012, p. 41) entende esse movimento como crucial na educação, pois os métodos tradicionais não condizem mais com as pessoas que cresceram jogando videogames por aproximadamente 20 anos. O autor complementa que o foco na gamificação aumenta o engajamento, a relevância e a imersão, e conduz a aprendizagem a um momento mais atual.

Mattar (2014, p. 43) apresenta preocupação semelhante e reforça a importância da inclusão dos jogos na educação, ao afirmar que:

\footnotetext{
Como educadores, precisamos procurar compreender como os designers de games conseguem atrair as pessoas para aprender games complexos, longos e difíceis. Os designers de games utilizam métodos eficientes para fazer as pessoas aprender e gostar de aprender, sendo, por isso, teóricos práticos do aprendizado. Precisamos então prestar atenção a bons jogos de computador e videogames, e aplicarmos os princípios de aprendizado que eles envolvem.
}

O propósito de utilizar elementos de jogos digitais especificamente no AVA tem origem na necessidade de transformar essas plataformas em ambientes mais interativos do que simples repositórios de conteúdo. Propiciar uma experiência diferenciada por meio da interface pode motivar e promover maior envolvimento dos alunos com o aprendizado. Pesquisas nesse sentido vêm sendo realizadas (FRANÇA, 2016; KLOCK, 2017).

Landers (2014), com o objetivo de construir uma teoria da aprendizagem gamificada para subsidiar projetos instrucionais, trabalha as definições de elementos de jogos digitais apresentada por Bedwell et al (2012), relacionando-os ao contexto da gamificação. O seu trabalho propõe que, para o estudo da gamificação, seria necessário aplicar esses elementos individualmente e realizar combinações para testar a eficácia e contribuir com resultados mais úteis à comunidade.

\subsection{Badges}

Um badge é um símbolo ou indicador de realização, habilidade, qualidade ou interesse. 
São emblemas que podem ser usados para reconhecer diferentes níveis de conhecimento, habilidades e comportamentos desenvolvidos pelos alunos, como, por exemplo: habilidade em se comunicar adequadamente, atitude colaborativa, proatividade e análise crítica. (MOZILLA, 2011).

Sua definição recorda a premiação com estrelinhas em atividades e avaliações no ensino fundamental. No entanto, os bagdes vêm sendo considerados como uma forma de certificação digital, comumente aplicados no reconhecimento de aprendizados não necessariamente relacionados ao ensino formal, mas em contextos diversos, levando em conta a aquisição de conhecimentos e habilidades além da sala de aula (GRECH; CAMILLERI, 2017). Já é possível acumular diferentes badges oriundos de diferentes experiências de aprendizagem em "mochilas" virtuais (MOZILLA, 2011), plataformas digitais que funcionam para os usuários concentrarem suas recompensas e divulgarem seus feitos a interessados do mercado de trabalho, de forma a complementar informações já divulgadas em currículos tradicionais.

Já no contexto formal de ensino, os badges vêm sendo aplicados para o reconhecimento de pequenas conquistas dos discentes ao longo de suas vidas acadêmicas e para incentivar determinados comportamentos no ensino superior (HAKULINEN; AUVINEN; KORHONEN, 2015).

\section{Disciplina de Metodologia da Pesquisa}

Um dos principais objetivos da disciplina é a elaboração de um projeto de pesquisa para ser desenvolvido posteriormente como Trabalho de Conclusão do Curso. Nesse percurso, há a necessidade de serem desenvolvidos espírito crítico, colaboração, estratégia e seguimento de regras como as da ABNT.

Em seus estudos, Sillaots (2014a, 2014b) buscou responder se a inclusão de elementos dos jogos digitais no processo de ensino e aprendizagem da disciplina de Metodologia Científica a tornaria mais agradável e aumentaria o engajamento dos alunos, que, segundo o autor, apresentam certa aversão à disciplina.

O estudo de caso realizado pelo autor teve origem na aplicação da gamificação em uma turma de mestrado do programa de gerenciamento de TI e Tecnologias Educacionais da modalidade presencial. A disciplina foi organizada como um jogo, com o objetivo de alcançar uma imersão mais profunda dos alunos, fazendo uso dos elementos: objetivos, avatar, pontos, scoreboard, níveis, sorte, colaboração, competição e feedback. 
Por meio de questionário no final da disciplina, a maioria dos alunos de Sillaots concordaram que os métodos de aprendizagem que requerem uma participação mais ativa são melhores que as apresentações passivas, e alguns sugeriram ainda a implantação de ainda mais elementos dos jogos digitais. Por outro lado, Sillaots concluiu que não é possível utilizar elementos que sejam igualmente motivadores para todos os alunos, e alguns deles chegam a não querer participar, mesmo que o objetivo seja aprender. Uma forma de minimizar isso é usar diferentes modos de jogar. Mesmo assim, o pesquisador pode concluir que o curso gamificado cria possibilidades de envolvimento ativo, e o fato de incluir a diversão em um ambiente sério mostrou-se para a maioria dos alunos como uma boa exceção em relação aos métodos tradicionais.

\section{Elaboração e Aplicação dos Badges}

Optou-se nesta pesquisa por usar apenas um elemento dos jogos, ou seja, a recompensa por badges, seguindo a sugestão de Landers (2014), com o intuito de melhor identificar o impacto do elemento aplicado na experiência dos discentes, sem sofrer a influência de outros elementos.

Norteando o planejamento, fez-se uso do template: "Badge System Design Worksheet'[1] (OPENBADGES, 2018) para conceber o conjunto de badges que fariam parte das recompensas dadas aos discentes. No planejamento, cada emblema foi associado a um tipo de atitude ou habilidade que se buscava encorajar nos discentes. A concepção gráfica foi realizada pelo ilustrador Eduardo Stumpf a partir das informações dispostas no template e discussões sobre cada um dos badges. Buscou-se utilizar uma linguagem gráfica única, com uma paleta de cores que fosse harmônica com o layout do AVA. Os badges se diferenciam entre si de acordo com a ilustração que busca simbolizar a conquista do aluno. No quadro 1 é exposto um resumo desse planejamento.

Quadro 1 - Planejamento dos badges 


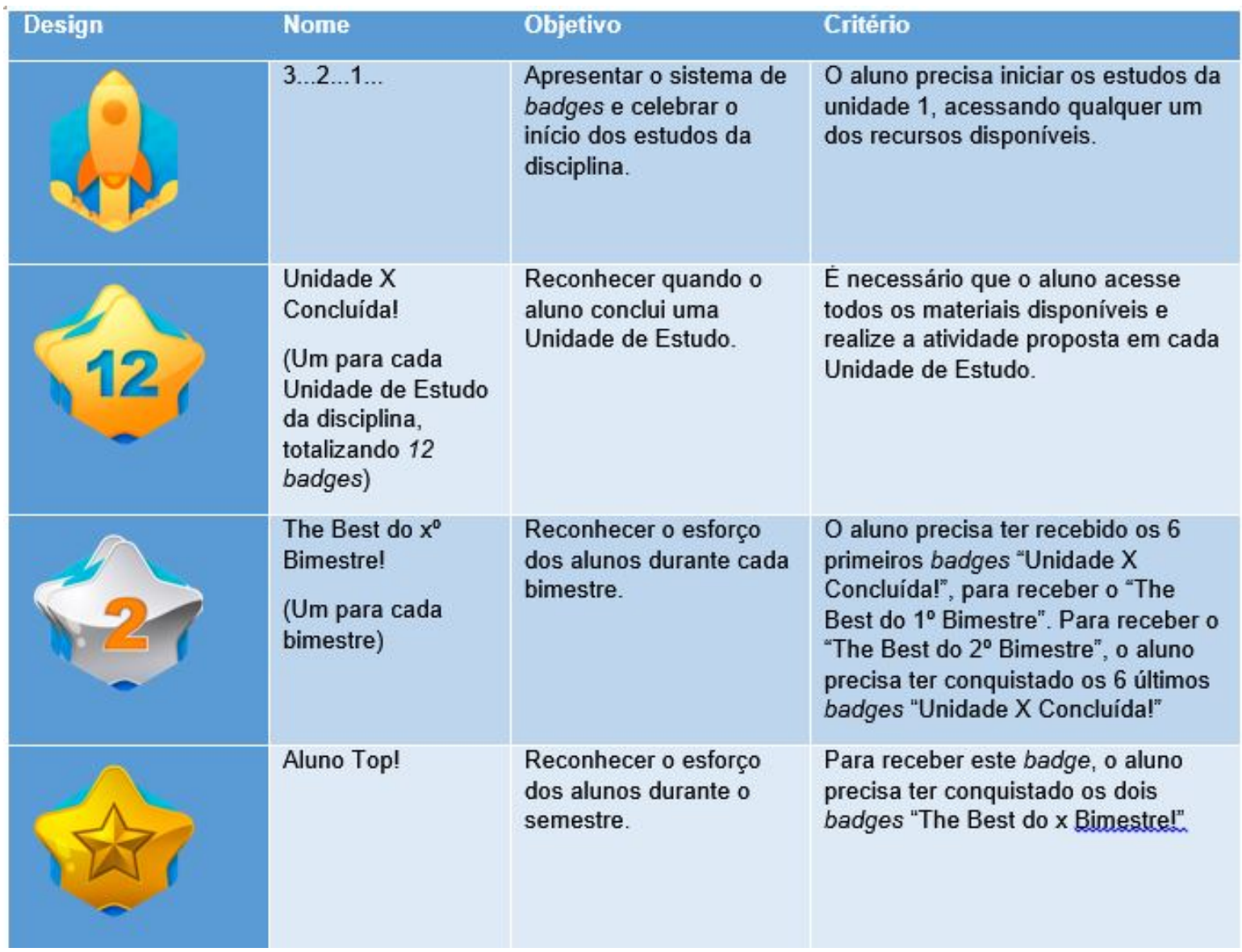
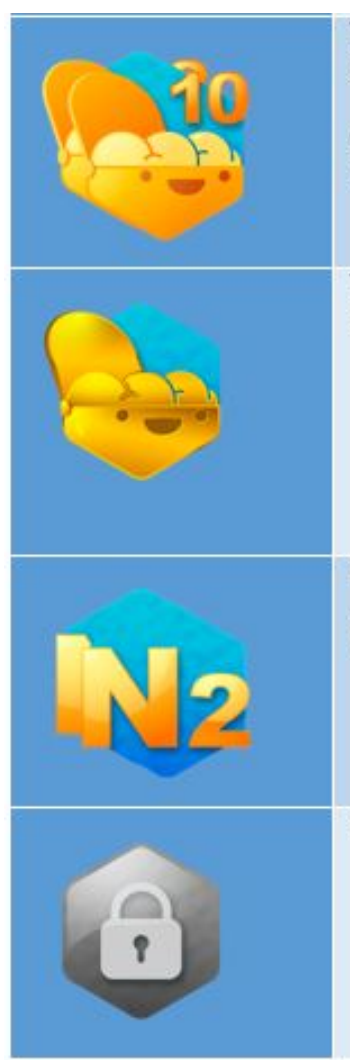

Teoria e Prática Unidade $\mathrm{X}$.

(Unidades 2, 4, $8 \mathrm{e}$ 10)

Top Teoria
Prática

Reconhecer o esforço do aluno ao realizar atividade que exige demonstração da relação da teoria apresentada na unidade com a prática.

Reconhecer a participação do aluno nos quatro fóruns que exigem demonstração da relação da teoria apresentada na unidade com a prática.

\begin{tabular}{l|l} 
N1 - Eu fui! & $\begin{array}{l}\text { Reconhecer a } \\
\text { participação do aluno na } \\
\text { avaliação presencial. }\end{array}$ \\
N2 - Eu fui! &
\end{tabular}
avaliação presencial.

Instigar a curiosidade do aluno por badges que ainda não estejam liberados.
$\mathrm{O}$ aluno é premiado o respectivo badge da unidade mediante publicação de mensagem nos fóruns específicos.

Para receber esse badge o aluno precisa ter participado de todos os 4 fóruns de teoria e prática.

O aluno precisa comparecer a cada avaliação presencial para receber cada um dos badges.

Nenhum aluno recebe esse badge mas todos conseguem visualizá-los acessando o gerenciamento de badges no dashboard. 
O AVA utilizado para a implementação da pesquisa foi o Moodle. Além de ser uma plataforma de código aberto, já permite a emissão de badges nos cursos por meio da ferramenta "Gerenciamento de badges". Ao adicionar um badge na plataforma, é necessário informar quais critérios os alunos terão que atender para recebê-lo. As opções de critérios incluem a conclusão de todo o curso mediante uma nota mínima, a conclusão de uma única atividade do curso ou várias, com ou sem prazo determinado para a realização.

Para este estudo, foram programados no AVA o recebimento das recompensas da seguinte forma: a partir do momento em que o discente atende os critérios estabelecidos para cada badge, recebe uma mensagem automática, felicitando-o e informando sobre a recompensa. Automaticamente também, o badge já é disponibilizado no seu perfil, conforme a Figura 1. Esses prêmios ficam visíveis para todos participantes da disciplina que visitam o perfil do discente.

Figura 1 - Visualização do quadro de badges pelo aluno

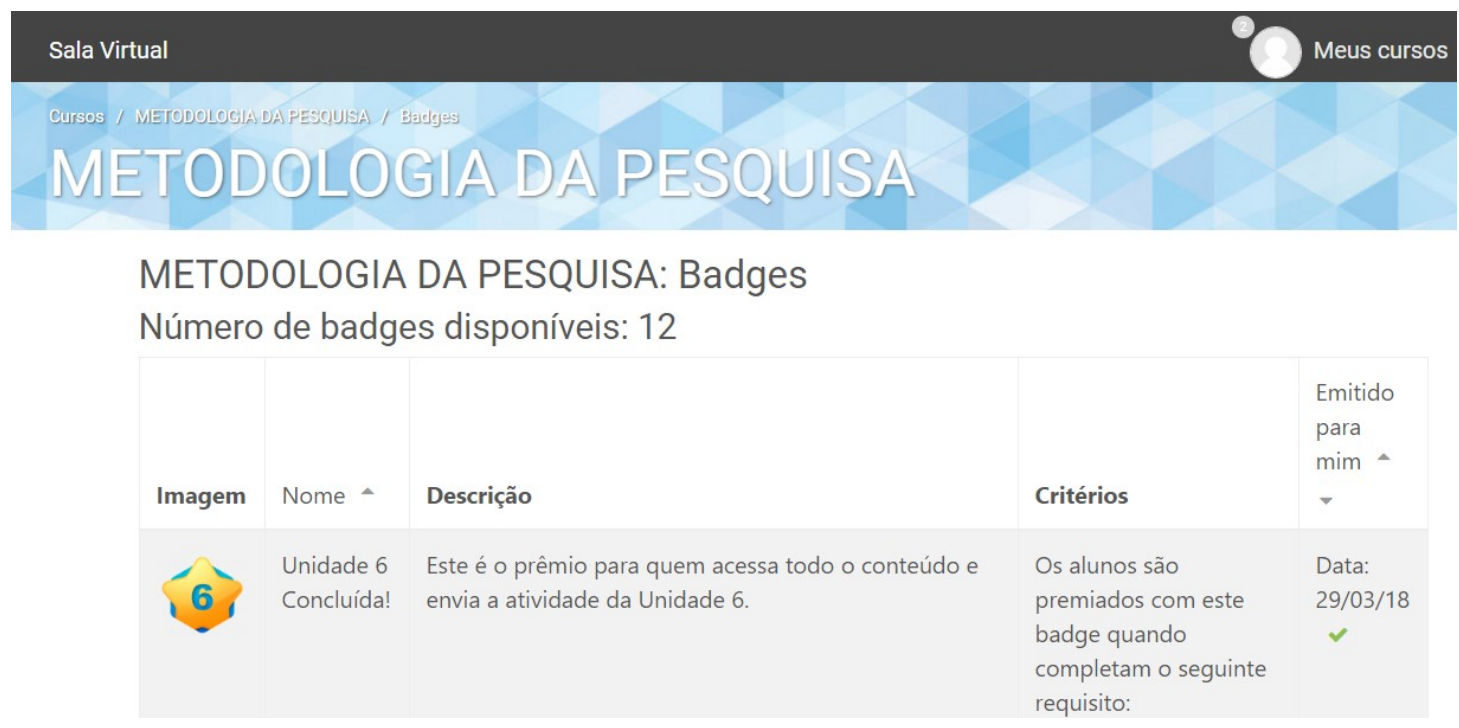

Fonte: Os autores

\section{Considerações Finais}

Apesar de ser uma pesquisa em andamento, é possível identificar algumas contribuições da implementação dos badges para o gerenciamento da disciplina. A partir do momento em que são criados critérios no Moodle para a emissão dos distintivos, relatórios sobre o comportamento dos alunos em relação a esses critérios ficam disponíveis na área de "Gerenciamento de Badges" da plataforma. Como os critérios podem ser determinados fazendo combinações diversas das atividades do curso, os 
dados gerados são diferentes dos relatórios já disponibilizados na plataforma. Esses dados permitem a realização de análises tanto em relação às recompensas, quanto às atividades necessárias para o seu recebimento. Por exemplo, é possível visualizar a quantidade de alunos que atenderam os critérios determinados e receberam as respectivas recompensas; também é possível identificar quais são os alunos que receberam cada um dos distintivos, as recompensas que foram mais e menos emitidas, entre outros.

Espera-se que o uso dos badges na disciplina promova maior engajamento e motivação dos discentes de metodologia da pesquisa na modalidade EaD.

\section{Referências}

BEDWELL, W. L. et al. Toward a Taxonomy Linking Game Attributes to Learning. Simulation \& Gaming, 2012.

DETERDING, S. et al. From game design elements to gamefulness. Proceedings of the 15th International Academic MindTrek Conference on Envisioning Future Media Environments - MindTrek '11. Anais. New York, New York, USA: ACM Press, 2011a. Disponível em: <http://dl.acm.org/citation.cfm?doid=2181037.2181040>. Acesso em: 15 jun. 2017

DETERDING, S. et al. Gamification. using game-design elements in non-gaming contexts. Proceedings of the 2011 annual conference extended abstracts on Human factors in computing systems - CHI EA '11. Anais. New York, New York, USA: ACM Press, 2011b.Disponível em: <http://portal.acm.org/citation.cfm?doid=1979742.1979575>. Acesso em: 10 jun. 2017

FRANÇA, R. Ambiente gamificado de aprendizagem baseada em projetos. 2016. Tese (Doutorado em Informática na Educação)_Universidade Federal do Rio Grande do Sul, Porto Alegre, 2016.

GRECH, A.; CAMILLERI, A. F. Blockchain in Education. Luxembourg: Publications Office of the European Union, 2017. Science for Policy report by the Joint Research Centre (JRC), the European Commission's science and knowledge service, edited by Andreia Inamorato dos Santos.

HAKULINEN, L.; AUVINEN, T.; KORHONEN, A. International journal of emerging technologies in learning. Kassel Univ. Press, 2015. v. 10. 
KAPP, K. M. The gamification of learning and Instruction. San Francisco: Pfeifer, 2012.

KLOCK, A. C. T. Análise Da Influência Da Gamificação Na Interação, Na Comunicação E No Desempenho Dos Estudantes Em Um Sistema De Hipermídia Adaptativo Educacional. 2017. Dissertação (Mestrado em Computação Aplicada)—Universidade do Estado de Santa Catarina, Joinville, 2017.

LANDERS, R. N. Developing a Theory of Gamified Learning. Simulation \& Gaming, v. 45, n. 6, p. 752-768, 15 dez. 2014.

MATTAR, J. Design Educacional: educação a distância na prática. 1. ed. São Paulo: Artesanato Educacional, 2014.

MOZILLA. Open Badges for Lifelong Learning. White Paper, p. 1-14, 2011.

OPENBADGES. Issuing Open Badges. Disponível em: <https://openbadges.org/getstarted/issuing-badges/>. Acesso em: 22 abr. 2018.

SILLAOTS, Martin. Achieving flow through gamification: a study on re-designing research methods courses. In: ECGBL - European Conference on Games Based Learning, 8., 2014, Berlin. BUSCH, Carsten (Ed.). Complete proceedings... Berlin: Academic Conferences and Publishing International Limited, 2014a. v. 2, p. 538-545.

SILLAOTS, Martin. Gamification of higher education by the example of course of research methods. In: International Conference on Web-Based Learning, 13., 2014, Tallinn, Estonia. POPESCU, Elvira et al (Ed.). Advances in web-based Learning-ICWL 2014. Springer International Publishing, 2014b. p. 106-115.

YIN, R. K. Estudo de caso. 5. ed. Porto Alegre: Bookman, 2015.

[1] Disponível em: https://docs.google.com/spreadsheet/ccc?key=0AnUOR-0CBMsTdE1 hWnJnYXINdnktVUIhWWdjVzFVR0E\&usp=drive_web\#gid =0 